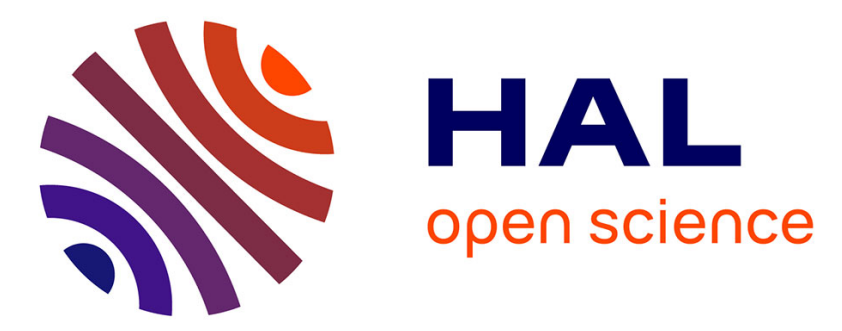

\title{
Lamellar eutectic growth with anisotropic interphase boundaries: Experimental study using the rotating directional solidification method
}

\author{
S Akamatsu, S Bottin-Rousseau, M Sererefoğlu, G S Faivre
}

\section{To cite this version:}

S Akamatsu, S Bottin-Rousseau, M Sererefoğlu, G S Faivre. Lamellar eutectic growth with anisotropic interphase boundaries: Experimental study using the rotating directional solidification method. Acta Materialia, 2012, 60 (6-7), pp.3206-3214. 10.1016/j.actamat.2012.02.033 . hal-01448370

\author{
HAL Id: hal-01448370 \\ https://hal.science/hal-01448370
}

Submitted on 16 Feb 2017

HAL is a multi-disciplinary open access archive for the deposit and dissemination of scientific research documents, whether they are published or not. The documents may come from teaching and research institutions in France or abroad, or from public or private research centers.
L'archive ouverte pluridisciplinaire $\mathbf{H A L}$, est destinée au dépôt et à la diffusion de documents scientifiques de niveau recherche, publiés ou non, émanant des établissements d'enseignement et de recherche français ou étrangers, des laboratoires publics ou privés. 


\title{
Lamellar eutectic growth with anisotropic interphase boundaries: Experimental study using the rotating directional solidification method
}

\author{
S. Akamatsu*, S. Bottin-Rousseau, M. Şerefoğlu** and G. Faivre \\ Institut des Nanosciences de Paris, UPMC, CNRS, 4 place Jussieu, 75252 Paris Cedex 05, France
}

\begin{abstract}
We report on an experimental study of the effects of interphase boundary anisotropy on eutectic microstructures using a new methodology called rotating directional solidification (RDS) that consists of rotating a thin sample with respect to a fixed unidirectional thermal gradient. The systems used are thin large eutectic grains of the $\mathrm{CBr}_{4}-\mathrm{C}_{2} \mathrm{Cl}_{6}$ and $\mathrm{In}-\mathrm{In}_{2} \mathrm{Bi}$ lamellar eutectic alloys. The shape of the observed RDS lamellar trajectories turns out to be a reproducible eutectic-grain dependent feature, in agreement with the theoretical prediction that these trajectories are approximately homothetic to the Wulff form of the interphase boundary in the sample plane. We show that different modes of lamellar growth ranging from quasi-isotropic to (crystallographically) locked exist in different eutectic grains of the two alloys studied. A detailed characterization of these modes is given, with particular attention to the as yet ill-understood aspects of locked lamellar growth.

Key words: Eutectic solidification, directional solidification, solidification microstructures, interphase boundaries
\end{abstract}

\section{Introduction}

The dramatic effects of a strong capillary anisotropy of interphase boundaries on eutectic directional-solidification patterns have long been noted [1, 2], but have

\footnotetext{
*Corresponding author

** Present address: Department of Mechanical Engineering, Koç University, Rumeli Feneri Yolu, 34450 Sariyer, Istanbul, Turkey

Email address: akamatsu@insp.jussieu.fr (S. Akamatsu )
} 
never been systematically studied. The property of the alloy that controls these effects is the Wulff plot of the interphase boundary in the reference frame of the laboratory. This Wulff plot varies from eutectic grain to eutectic grain, where the term "eutectic grain" (EG) designates both a given interphase orientation relationship - i.e. a heterophase bicrystal - and a given orientation of this bicrystal with respect to the directional-solidification setup. Careful experimentation requires a good crystallographic homogeneity of the samples, i.e. large-sized eutectic grains, and a small number of free orientational parameters. The use of thin-sample directional solidification, in addition to reducing the dimensionality of the solidification pattern, allows one to grow nearly perfect large-sized eutectic grains [3]. The use of a rotating directional solidification (RDS) stage whereby a thin sample is rotated with respect to a fixed unidirectional thermal gradient, moreover permits to vary a single orientational parameter in situ while keeping the other parameters constant.

In this article, we present experimental observations performed by RDS in the $\mathrm{CBr}_{4}-\mathrm{C}_{2} \mathrm{Cl}_{6}$ and In- $\mathrm{In}_{2} \mathrm{Bi}$ lamellar eutectics. More specifically, we study the RDS lamellar patterns (i.e. the forms of the RDS lamellar trajectories) in a series of different eutectic grains in both alloys. Each RDS pattern turned out to be a reproducible feature of the EG to which it belonged, indicating that RDS patterns only depend on the characteristics of the Wulff plot of the interphase boundary. All the observed RDS patterns could be grouped into three categories with contrasting characteristics (quasi-isotropic, nearly-locked, locked) independently of the alloy. We show that all the observations can be simply explained by means of the socalled "symmetric-pattern approximation". This approximation will be discussed in detail in another article. It can be summed up as follows. Based on general principles, an interphase boundary anisotropy is expected to break the spatiotemporal symmetry (symmetry with respect to the growth axis $\mathbf{z}$ and time-invariance) distinctive of isotropic lamellar patterns. In other words, lamellar patterns should have asymmetric forms of their solid-liquid interfaces and travel laterally along the growth front (entailing that the lamellae left behind in the solid are inclined with respect to $\mathbf{z}$ ) in the presence of a strong interphase boundary anisotropy. However, on closer examination, it can be seen that the steady-state travelling velocity $v_{s t}$ of the patterns must be such that the solid-liquid interfaces remain nearly symmetric. The symmetric-pattern approximation consists of assuming that $v_{s t}$ is such that the solid-liquid interfaces are exactly symmetric, or, equivalently, that the surface tension force $\sigma$ of the interphase boundaries is parallel to $\mathbf{z}$ (Fig. 1) -see Ref. [4] for a definition of $\sigma$. As regards RDS experiments, it can be demonstrated that the symmetric-pattern approximation implies that the trajectories of 
the lamellae in the sample reference frame are homothetic to the 2D Wulff form of the interphase boundary in the EG under consideration. Thus, for instance, RDS lamellar trajectories should be almost circular in EGs where the interphase boundary anisotropy is negligible, and faceted in EGs where the Wulff plot of the interphase boundary has cusp singularities (epitaxial EGs). This is the only consistent explanation of the familiar phenomenon of crystallographic locking of lamellar patterns. The report also includes a detailed description of the as-yet unknown dynamical features of (crystallographically) locked lamellar patterns.

\section{Experimental methods}

\subsection{Alloys and crucibles}

The $\mathrm{CBr}_{4}-\mathrm{C}_{2} \mathrm{Cl}_{6}$ alloy has a eutectic plateau at $T_{E}=84.4^{\circ} \mathrm{C}$. The concentration of the eutectic liquid is $C_{E}=11.6 \mathrm{~mol} \% \mathrm{C}_{2} \mathrm{Cl}_{6}$. The eutectic phases are a fcc phase $(\alpha)$ and a bcc phase $(\beta)$ with concentrations 8.8 and $18.5 \mathrm{~mol}_{2} \mathrm{C}_{2} \mathrm{Cl}_{6}$, respectively $[1,5]$. The $\mathrm{In}-\mathrm{In}_{2} \mathrm{Bi}$ alloy has a eutectic plateau $\left(T_{E}=72.7^{\circ} \mathrm{C}\right.$, $C_{E}=22.2 \mathrm{at} \% \mathrm{Bi}$ ) between a fct phase $(\epsilon)$ of concentration $11.5 \mathrm{at} \% \mathrm{Bi}$ and the hexagonal $\mathrm{In}_{2} \mathrm{Bi}$ intermetallic compound [6]. The value of the Jackson-Hunt (JH) constant $K_{J H}=\lambda_{\mathrm{JH}} V^{0.5}$ at eutectic concentration, where $\lambda_{\mathrm{JH}}$ is the minimumundercooling spacing [1], was estimated to be $13.8 \pm 0.5 \mu \mathrm{m}^{1.5} \mathrm{~s}^{-0.5}$ in $\mathrm{CBr}_{4}-\mathrm{C}_{2} \mathrm{Cl}_{6}$ [7], and $7.07 \pm 0.35 \mu \mathrm{m}^{1.5} \mathrm{~s}^{-0.5}$ in $\mathrm{In}-\mathrm{In}_{2} \mathrm{Bi}$ [8]. Both $\mathrm{CBr}_{4}-\mathrm{C}_{2} \mathrm{Cl}_{6}$ and $\mathrm{In}-\mathrm{In}_{2} \mathrm{Bi}$ undergo a eutectoid transformation between $T_{E}$ and room temperature rendering post-mortem orientation measurements by $\mathrm{X}$-ray diffraction problematic. We used nominally eutectic (in fact, slightly hypereutectic) alloys. The $\operatorname{In}_{-} \operatorname{In}_{2} \mathrm{Bi}$ alloy was prepared from $99.999 \%$ pure indium and bismuth (Goodfellow), and the $\mathrm{CBr}_{4}-\mathrm{C}_{2} \mathrm{Cl}_{6}$ alloy from sublimated compounds. Thin crucibles were made of two $0.3 \mathrm{~mm}$-thick glass plates separated by $12 \mu \mathrm{m}$-thick plastic strips or $13 \mu \mathrm{m}$-diameter tungsten wires. It has been checked that convection flows in the liquid were suppressed in samples of this thickness. The in-plane dimensions of the rectangular space available for solidification were of $15 \times 30 \mathrm{~mm}^{2}$. The crucibles were filled with molten alloys by capillarity $\left(\mathrm{CBr}_{4}-\mathrm{C}_{2} \mathrm{Cl}_{6}\right)$ or by a vacuum suction method $\left(\mathrm{In}-\mathrm{In}_{2} \mathrm{Bi}\right)$, and then sealed at room temperature. Residual impurities contained in our samples induced the formation of eutectic cells at pulling velocities higher than $5 \mu \mathrm{ms}^{-1}$ in both alloys. The solidification rates considered herein are well below this threshold value. 


\subsection{Method of observation}

The growth front was observed in real-time in the $\mathbf{y}$ direction with an inverted optical microscope. The transparent $\mathrm{CBr}_{4}-\mathrm{C}_{2} \mathrm{Cl}_{6}$ eutectic was observed in transmitted light. With this method, the contrast originates from the differences in refrative index of the different thermodynamic phases and reveals the interfaces. The In- $\mathrm{In}_{2} \mathrm{Bi}$ eutectic was observed in reflected light through the lower glass wall of the crucible [9]. The differences in reflectivity between the different types of surfaces of contact gave rise to a contrast, which, after numerical enhancement, made the $\epsilon$-glass surfaces appear white, the $\mathrm{In}_{2} \mathrm{Bi}$-glass surfaces black, and the liquid-glass surfaces grey. Perturbations (contrast effects making the $\epsilon$-glass contacts appear grey instead of white, barbules formed at the rear of the growth front) due to complex wetting and segregation phenomena at the sample walls [8] can be seen in some places in the micrographs, but are of no importance for our present purpose. Images were recorded with a monochrome digital camera, and transferred to a PC for further analysis. A several millimeter-wide region of interest was regularly scanned using the motorized stage of the microscope.

\subsection{The rotating directional solidification stage}

A RDS stage was built based on a model introduced by Oswald et al. [10] (Fig. 2). It comprises: (i) a thermal-gradient bench composed of two thermally regulated copper blocks delimiting a $5 \mathrm{~mm}$-wide observation slot; (ii) a rectangular housing sliding on top of the copper blocks along z; (iii) a $15 \mathrm{~cm}$-diameter circular plate, pierced by a rectangular sample-holding cavity, inserted in the rectangular housing, and rotatable about the $\mathbf{y}$ axis. Small clamps mildly press the sample on the copper blocks to ensure both good thermal contact and smooth sliding motion. The position of the growth front along $\mathbf{z}$ is monitored over time to ensure that the stability of the temperature distribution is satisfactory. Slight deviations of the thermal field from unidirectionality due to the geometry of the setup occurred during RDS. However, the $T_{E}$-isotherm remained rectilinear inside the region of interest, so that these deviations were equivalent to small variations of angular speed over time, and therefore did not affect the RDS lamellar trajectories. The translation speed is denoted by $V_{T}$, and the angular speed by $\omega=d \theta / d t$, where $t$ is the time and $\theta$ is the angle between $\mathbf{z}$ and the axis of the sample. The $\omega$ values used

were at most $5 \times 10^{-4} \mathrm{rads}^{-1}(\approx 0.3 \mathrm{rph})$. The thermal gradient at the solid-liquid interface was $100 \pm 10 \mathrm{Kcm}^{-1}$. 


\subsection{Experimental protocol}

The experiments were conducted as follows (Fig. 3). A solidified sample is inserted into the experimental setup in a tilted position and pushed toward the hot block until the solid is reduced to a small seed polycrystal (Fig. 3a). The sample is then submitted to a EG-selection process. At the end of this process, the sample is directionally solidified at a constant $V_{T}$ value until the center of rotation $\Omega$ approximately sits on the growth front (Fig. 3b). At this moment, which is taken as zero time point, the pulling is stopped, and a RDS-run at constant $\omega$ is started (Fig. $3 \mathrm{c})$. The position $R$ of the growth front with respect to $\Omega$ was less than $100 \mu \mathrm{m}$ in all the experiments. It could not be measured with accuracy at this stage of the experiment, but was determined later by a method which is explained below. The RDS-run is then pursued during periods which can cover several complete revolutions of the sample. Although the grown microstructure is destroyed by melting after a half turn, an uninterrupted recording of the growth front allows one to perform retroactively a mosaic reconstruction of the whole solidification process in a reference frame attached to the sample. These reconstructed microstructures are, of course, multiple-valued functions of $\theta$ for RDS-runs covering more than a revolution of the sample, and have to be represented by a set of reconstructions limited to one revolution (thereafter called "space-time maps") containing a radial cut.

Particular requirements were met during this study. The duration time of the pulling at constant $V_{T}$ was sufficiently long for a quasi steady lamellar pattern, which served as an initial condition for the subsequent RDS-run, to be formed. Out-of-plane locked lamellar patterns were identified in situ and eliminated from the study in the case of $\mathrm{CBr}_{4}-\mathrm{C}_{2} \mathrm{Cl}_{6}$ samples. The EG-selection process was performed in either of the two ways: (i) an epitaxial EG that preexisted in the seed (and was probably formed by nucleation of one phase onto the other during the solid-solid phase transition occurring between room temperature and $T_{E}$ ) was allowed to expand laterally, or (ii) the preexisting EGs were overgrown through a process called lateral invasion [3], which led to the formation of a few millimeterslarge EGs with more or less random interphase orientation relationships thereafter called random EGs.

\section{Experimental results}

Several epitaxial and random EGs were studied by RDS in $\mathrm{CBr}_{4}-\mathrm{C}_{2} \mathrm{Cl}_{6}$ and $\mathrm{In}-\mathrm{In}_{2} \mathrm{Bi}$. The space-time maps recorded in these EGs can be classified into a small number of categories (quasi-isotropic, nearly locked, locked) independently of the alloy concerned. Representative examples of each category are studied 
below. Most importantly, all of the studied space-time maps were essentially $\pi$ periodic and reproducible from turn to turn, regardless of their complexity. This establishes that the space-time map of a RDS experiment reveals a characteristic feature, called "RDS pattern", of the EG to which it belongs. As mentioned above, our working hypothesis is that the lamellar trajectories making up an RDS pattern are homothetic to the 2D Wulff form of the interphase boundary in the EG studied.

\subsection{Quasi-isotropic RDS patterns}

Quasi-isotropic RDS patterns - i.e. RDS patterns, in which lamellar trajectories remained nearly perpendicular to the growth front throughout the RDS-run - were typical of random EGs in both $\mathrm{CBr}_{4}-\mathrm{C}_{2} \mathrm{Cl}_{6}$ and $\mathrm{In}-\mathrm{In}_{2} \mathrm{Bi}$ (Fig. 4). Let us first of all consider the question of the time-evolution of the $\lambda(x)$ distribution in these patterns. It is generally known that the dynamics of isotropic lamellar patterns (also called "floating" dynamics) is essentially controlled by one variable, the reduced spacing $\Lambda=\lambda / \lambda_{\mathrm{JH}}=\lambda V^{1 / 2} / K_{J H}$. During standard directional solidification, this dynamics includes (i) a process of relaxation toward regularity called $\lambda$-diffusion, which occurs inside a certain stability interval, and (ii) instability mechanisms occurring at the bounds of this interval. These bounds are typically located at $\Lambda_{1}=0.8$ and $\Lambda_{2}=2.2$ at eutectic concentration, the former value corresponding to the onset of a lamella elimination instability and the latter to an oscillatory bifurcation $[11,12]$. During RDS, the solidification velocity varies according to $V=\rho \omega$, where $\rho$ is the position along the growth front measured from $\Omega$. Thus, under a local-dynamics assumption which is generally valid in lamellar eutectic growth, the stability bounds of a lamellar pattern during RDS are located at the positions $\rho_{i=1,2}$ given by $\Lambda_{i}=\lambda\left(\rho_{i} \omega\right)^{1 / 2} / K_{J H}$. In other words, a lamellar pattern with a uniform spacing at $t=0$ should remain stable in the region of the growth front extending from $\rho_{1}$ to $\rho_{2}$ and undergo the above-mentioned instabilities outside this region.

The experimental observations clearly support this conjecture. Large-scale modulations of $\lambda$ inherited from the zero-time state were observed to remain unchanged throughout the RDS-runs in agreement with the fact that the $\lambda$-diffusion times over the ranges of the modulations were long compared to experiment durations. In Fig. 4a, for instance, $\lambda$ ranged from 16.4 to $17.6 \mu \mathrm{m}, V$ ranged from 0.58 to $0.93 \mu \mathrm{ms}^{-1}$, and $\Lambda$ ranged from 0.90 to 1.26 . In Fig. $4 \mathrm{~b}, \lambda$ ranged from 14.4 to $11.5 \mu \mathrm{m}, V$ ranged from 0.35 to $0.54 \mu \mathrm{ms}^{-1}$, and $\Lambda \approx 1.2$ over the field of view. In both cases, the $\lambda$ distribution fell well within the stability interval, and did not significantly evolve over time. Still in agreement with the above considerations, 
the centre of the sample functioned as a lamellar sink as a result of the inwardbound instrumental drift linked to the fact that $\Omega$ sat slightly above the growth front in these experiments (see below). In Figure 5, which comes from the same experimental run (RDS1) as Fig. 4a, a lamella elimination event is seen to occur at $\rho$ close to the calculated value of $\rho_{1}(850 \mu \mathrm{m})$. A central region of radius about $\rho_{1} / 3$ is occupied by a $\beta$ single crystal, the solid-liquid interface of which assumes a stationary sigmoid shape going from the temperature of the $\beta$ liquidus on the melting side of the sample to $T_{E}$ on the growing side. No perturbation linked to this local structure was observed beyond the central region.

A space-time map from Run RDS1 is shown in Figure 6. It can be seen that the RDS lamellar trajectories are not circles, but spirals approaching the central region of the sample. This is an instrumental effect due to the non-zero distance of the growth front to $\Omega$ in this experiment. In such a case, the growth front remains tangent to the circle of radius $R$ and centre $\Omega$ throughout the experimental run, and the orthogonal trajectories to the growth front - which, in isotropic systems, represent the RDS lamellar trajectories - are involutes of this circle. Moreover, the direction of travel along the circle involutes is centripetal when $R<0$, which was the case for Run RDS1, and centrifugal when $R>0$. We performed leastsquare fittings of the equation of a circle involute to lamellar trajectories taking $R$ as adjustable parameter. The best fit was obtained for $R=-70 \pm 10 \mu \mathrm{m}$ for all the trajectories studied. A $\pi$-periodic smoothing of the fitting residuals gave practically identical results for two widely $(\approx 50 \lambda)$ apart lamellae and showed that the measured lamellar trajectories significantly deviated from the calculated ones by about $10^{-2}$ rad (see Inset). Whether this deviation was due to weak interfacialanisotropy effects (including interphase boundary anisotropy and, possibly, solidliquid interface anisotropy effects) or some instrumental imperfection could not be ascertained. Similar results were obtained in other quasi-isotropic EGs. Thus, the experimental uncertainty (excluding the errors of unknown magnitude pertaining to the symmetric-pattern approximation) on the 2D Wulff plots determined by our experiments was at most of $1 \%$. This is a minor error compared to the anisotropy effects studied in the remainder of this article. Altogether, these observations substantiate the earlier assumption of a quasi-isotropic lamellar growth in some random EGs of $\mathrm{CBr}_{4}-\mathrm{C}_{2} \mathrm{Cl}_{6}$ and $\mathrm{In}-\mathrm{In}_{2} \mathrm{Bi}[11,8]$.

\subsection{Nearly locked RDS patterns}

In some EGs, we observed smooth-looking oval lamellar trajectories, indicative of a floating dynamics coexisting with a pronounced minimum in the $2 \mathrm{D}$ Wulff plot. The $\lambda(x)$ distributions underwent dilatation-compression cycles linked 
to the oval shape of the lamellar trajectories while remaining essentially uniform at each time point, confirming the presence of an effective $\lambda$-diffusion. These RDS patterns were therefore called "nearly locked". An example from a run performed in $\mathrm{In}-\mathrm{In}_{2} \mathrm{Bi}$ (Run RDS2) is given in Figure 7. The analysis of this example proceeded as follows. The inclination angle $\phi$ of the lamellae at the growth front was measured point by point over a sufficiently broad $\theta$ range. Different lamellae yielded identical data within experimental error. The Wulff plot $\gamma(\phi)$ was calculated from the $\phi(\theta)$ data by integrating the differential equation that relates these two quantities [4]. Various $\pi$-periodic smoothing functions were tentatively adjusted onto the $\gamma(\phi)$ data. A good fit was achieved with the function $\gamma(\phi)=1-\epsilon_{c} \exp \left[-\left(\phi / w_{c}\right)^{2}\right]-\epsilon_{2} \cos (2 \phi)-\epsilon_{4} \cos (4 \phi)$, where $\epsilon_{c}=0.0507$, $w_{c}=0.195, \epsilon_{2}=0.0854, \epsilon_{4}=0.0221$, the origin of $\phi$ being taken at the minimum of $\gamma(\phi)$ (Fig. 7). The 2D Wulff plot thus determined exhibits a relatively strong mostly twofold anisotropy and a trough with quantitative features (a depth of $\approx 5 \%$ and an angular aperture of $\approx 10^{\circ}$ ) comparable to those found for cusps corresponding to low-energy orientations of heterophase boundaries in various materials [13]. To sum up, Run RDS2 is a representative example of EGs with a strong non-singular (i. e. devoid of cusp-like minima and forbidden angular ranges) interphase boundary anisotropy.

\subsection{Locked RDS patterns}

The contrasting characteristics (travelling vs. steady, irregular vs. regular) of the locked and floating types of lamellar patterns in epitaxial and random EGs, respectively, are illustrated in Fig. 8. The two displayed micrographs were taken during standard (without rotation) directional solidification in thin $\mathrm{CBr}_{4}-\mathrm{C}_{2} \mathrm{Cl}_{6}$ (Fig. 8a) and $\mathrm{In}-\mathrm{In}_{2} \mathrm{Bi}$ (Fig. 8b) samples. The similarity between either type of lamellar pattern in the two alloys should also be noted.

Let us now turn to RDS patterns. Most epitaxial EGs exhibited RDS patterns indicative of deep cusps with forbidden vicinal angular ranges in the Wulff plots of the interphase boundary. The space-time maps observed in these EGs showed a great geometrical variety, as could be expected from the large number of orientational degrees of freedom of the EGs. Broadly speaking, however, they all consisted of a succession of different locking or floating angular ranges, separated by transition angular ranges, exhibiting, in general, sawtooth lamellar trajectories. Figure 9 shows an example coming from a run performed in $\mathrm{In}_{-} \mathrm{In}_{2} \mathrm{Bi}$ (Run RDS3), which comprised eleven revolutions of the sample that yielded eleven almost identical space-time maps. During this run, the instrumental drift was centrifugal so that, after a few turns, the whole solid originated from the central source and was 
therefore certain to be a single EG. Globally, Fig. 9 discloses three main angular ranges separated by transition angular ranges of variable widths. A closer examination reveals an unexpected complexity of the growth dynamics in each of them.

The following aspects of locked RDS patterns deserve special attention:

1- Crystallographic locking. Snapshots taken near the two endpoints of a locking angular range during a run called RDS4 are shown in Figure 10. Each of the two snapshots shows a very irregular, essentially time-independent $\lambda(x)$ distribution. In this example, the local $\lambda$ values ranged from about 5 to $30 \mu \mathrm{m}$, and $\Lambda$ from about 0.5 to 3 , which means that most of the lamellae were well outside the stability range of isotropic lamellar patterns and remained nevertheless steady. Incidentally, the $\Lambda$ values that have just been mentioned were calculated taking into account the fact that $\lambda_{\mathrm{JH}}$ decreases as the contact angles decrease, and is thus smaller in locked than in floating lamellar patterns, everything else equal, which led us to $\lambda_{\mathrm{JH}}=10 \pm 1 \mu \mathrm{m}$ in this example. Furthermore, Fig. 10 shows that the rectilinear trajectories of the locked interphase boundaries are perturbed by a sporadic formation of notches of amplitude usually much smaller than $\lambda$, which sometimes leads to branching events. This is not understood at all at present. The segments of interphase boundary extending between successive notches appear perfectly straight and parallel to each other within experimental error. During Run RDS4, their inclination angle was measured to be a linear function of $\theta$ with slope unity over a $\theta$-range of more than $45^{\circ}$. Thus, these segments were undoubtedly locked onto a fixed crystallographic direction.

2- Transition angular ranges. A view of sawtooth RDS lamellar trajectories observed in $\mathrm{In}_{-} \mathrm{In}_{2} \mathrm{Bi}$ (Run RDS5) is shown in Figure 11. These trajectories are composed of relatively long locked and unlocked segments separated by sharp edges, indicating that the angular range under consideration is a forbidden range for the interphase boundary. The unlocked segments do not seem to correspond to a definite floating state of the system, but are rather part of a nonlinear oscillatory mode of the lamellar pattern. This view is further supported by the periodicity in space and time assumed by the locking-unlocking process in some places. Solitary waves sweeping large portions of the growth front bring about an additional complexity. This complex dynamics should be studied by standard directional solidification in the future.

3- Multistability. A coexistence of distinct lamellar patterns along the growth front can be seen in each of the main angular ranges of Fig. 9. A definitive identification of the corresponding metastable branches of lamellar states would require metallographic data about the $3 \mathrm{D}$ orientation of the lamellar planes, which 
are not available. The following remarks are therefore somewhat conjectural. Of the branches displayed in Fig. 9, two ( $1 a$ and $3 a)$ are visibly floating, and the others locked. The floating patterns $1 a$ and $3 a$ could plausibly be occurrences of a single 2D nearly locked branch similar to the one studied in Section 3.2. The locked patterns $1 b, 2 a$ and $3 b$ could also correspond to $2 \mathrm{D}$ branches. In contrast, the $2 b$ locked pattern, which covers essentially the same broad $\left(\approx 65^{\circ}\right)$ angular range as $2 a$ (and is moreover separated from $1 a$ by a very discontinuous transition process), is likely to be an out-of-plane pattern. This illustrates the relatively frequent occurrence of metastability between different kinds of locked and floating lamellar patterns in RDS of epitaxial EGs.

\section{Conclusion}

This study has shown that the form of lamellar trajectories yielded by rotating directional solidification (RDS pattern) is a reproducible, eutectic-grain specific feature, which has a crystallographic origin. The hypothesis that a RDS patterns is approximately homothetic to the Wulff form of the interphase boundary in the sample plane was found to be compatible with most of the experimental observations. Numerical studies will be needed to evaluate the error committed using this approximation.

The link between 2D Wulff plots acquired by RDS and interphase orientation relationships in the EGs is not yet fully clarified. Three distinct categories (quasiisotropic, nearly locked, locked) of RDS patterns have been highlighted during this study. A locked RDS pattern unambiguously indicates an epitaxial EG, i.e., an EG where the interphase boundary has singular low-energy orientations. Quasiisotropic RDS patterns, which were observed in more or less randomly oriented EGs, are likely to originate from EGs with quasi-isotropic 3D Wulff plots. Nearly locked RDS patterns pose a somewhat more complex, and particularly interesting, question. There are basically two possibilities. A EG, in which a nearly locked RDS patterns is observed, may be epitaxial in the strict sense, but oriented in such a way that the singularities of the 3D Wulff plot are outside the sample plane the smooth trough in the 2D Wulff plot corresponding to a smooth region of the depression surrounding a 3D singularity. Or the EG itself may be only pseudoepitaxial, meaning that the surface tension minima of the interphase boundary are smooth. X-ray and EBSD studies would be necessary to clarify this point. In any case, the discovery of nearly locked RDS patterns during this study demonstrated that a steep inclination of the lamellae with respect to the growth direction is not necessarily associated with a dynamical locking (i.e. an absence of $\lambda$-relaxation 
mechanism), but can coexist with a floating dynamics. The inclined but nevertheless essentially regular lamellar patterns that have been observed in some Al-based eutectics [14] might belong to the nearly-locked category.

\section{Acknowledgments}

We are greatly indebted to C. Picard, who drew the plans of, and built the RDS setup. We warmly thank P. Ott for her help in the preparation of the samples. We gratefully acknowledge numerous fruitful discussions with M. Plapp, U. Hecht, S. Rex and V. Witusiewicz. We also thank J. Van Suchtelen for communicating inspiring excerpts from an unpublished RDS investigation of lamellar eutectic growth. One of us (MS) was supported financially by the Centre National d'Etudes Spatiales, France.

\section{References}

[1] Hunt JD, Jackson KA. Trans Metall Soc AIME 1966;236:1129.

[2] Hogan LM, Kraft RW, Lemkey FD. Adv. Mater. Res. 1971;5:8.

[3] Akamatsu S, Moulinet S, Faivre G. Metall Mater Trans A 2001;32:2039.

[4] Hoffmann DW, Cahn J.W. Surf Science 1972; 31:368.

[5] Mergy J, Faivre G, Guthmann C, Mellet R. J Cryst Growth 1993;134:353.

[6] Massalsky TB, Subramanian PR, Okamoto H, Kasprzak L. Binary Alloys Phase Diagram, 2nd Ed. Materials Park, ASM Inst.

[7] Akamatsu S, Plapp M, Faivre G, Karma A. Metall Mat Trans A 2004;35:1815.

[8] Akamatsu S, Bottin-Rousseau S, Faivre G. Acta Mater 2011;59: 7586.

[9] Rex S, Witusiewicz V, Hecht U. Mater Sci Engr A 2005;413-414:249.

[10] Oswald P, Moulin M, Metz P, Géminard JC, Sotta P, Sallen L. J. Phys. France III 1993;3: 1891.

[11] Ginibre M, Akamatsu S, and Faivre G. Phys. Rev. E 1997;56:780.

[12] A. Karma and A. Sarkissian, Met. Trans. A 1996 ; 27: 635 
[13] Sutton A.P., Balluffi R.W. Interfaces in Crystalline Materials ; Oxford University Press; 2009.

[14] Hecht U, Witusiewicz VT, Drevermann A, Rex S. Trans. Indian Inst. Met. 2005;58:545 
Figure 1: Repeat unit of a lamellar eutectic pattern in a system with a strong interfacial anisotropy of the interphase boundary according to the symmetric-pattern approximation. $\alpha, \beta$ : eutectic phases. $L$ : liquid. z: direction of the thermal gradient (growth direction). $\mathbf{y}$ : normal to the sample plane. $\mathbf{x}$ : average direction of the solid-liquid interface. $\phi$ : inclination angle. $\sigma$ : surface tension force of the interphase boundary. $\gamma_{\alpha}$ and $\gamma_{\beta}$ : surface tension forces of the supposedly isotropic solid-liquid interfaces.

Figure 2: Technical sketch of the RDS stage. P: sole plate fixed on the translation stage of the microscope. $\mathrm{T}-$ and $\mathrm{T}+$ : cold and hot blocks fixed on the sole plate. $\mathrm{H}$ : housing. W: sprocket wheel on the periphery of a rotatable circular plate. M1: translation motor. M2: rotation motor. S: worm screw. $V_{T}$ : translation speed. $\omega$ : angular speed. xyz: laboratory reference frame.

Figure 3: Protocol for an RDS experimental run. (a) Seed crystal and eutectic-grain selection; (b) Standard directional solidification; (c) Start of RDS; (d) Long-duration RDS. Cross: centre of rotation $(\Omega)$. Empty rectangles: hot and cold blocks of the thermal bench.

Figure 4: Snapshot taken during RDS experimental runs displaying quasi-isotropic RDS patterns. The centre of rotation was located outside the field of view, slightly above the growth front, at a distance of about $1.2 \mathrm{~mm}$ from the left edge of the images. a) $\mathrm{CBr}_{4}-\mathrm{C}_{2} \mathrm{Cl}_{6} ; \omega=5.0 \times 10^{-4} \mathrm{rad} \mathrm{s}^{-1}$ (Run RDS1). The thicker lamellae are $\alpha$ and the thinner ones $\beta$. Inset: enlarged view (width: $100 \mu \mathrm{m})$. b) $\operatorname{In}_{-} \operatorname{In}_{2} \mathrm{Bi} . \omega=2.5 \times 10^{-4} \mathrm{rad} \mathrm{s}^{-1}$. The $\epsilon$ phase appears white and $\operatorname{In}_{2} \mathrm{Bi}$ black. Bars: $100 \mu \mathrm{m}$.

Figure 5: Snapshot of the central region during Run RDS1. Cross: calculated position of $\Omega$. Bar: $100 \mu \mathrm{m}$.

Figure 6: Space-time map from Run RDS1. The direction of growth is counterclockwise. Straight line: radial cut. For the blank central area, see Fig. 5. The rare lamella termination events that occurred inside the stability range were due to extraneous particles being pushed along by the growth front. L1, L2: best-fitting circle involutes. Image size: $3.675 \times 3.675 \mathrm{~mm}^{2}$. Inset: Residual plots of the best-fitting circle involutes. Curves: smoothed curves through the residuals.

Figure 7: Top: Space-time map over a $\pi / 2$ angular range of a nearly locked lamellar pattern (Run RDS2). In- $\mathrm{In}_{2}$ Bi eutectic. $\omega=2.5 \times 10^{-4} \mathrm{rad} \mathrm{s}^{-1}$. Arrow: growth direction. For easy readability, the image has been binarized and every other $\operatorname{In}_{2} \mathrm{Bi}$ lamella has been removed. Bar: $100 \mu \mathrm{m}$. Inset: $\phi(\theta)$ function measured along the two emphasized lamellae. Bottom: Calculated 2D Wulff form and 2D Wulff plot of the interphase boundary. Symbols: point-by-point calculation. Continuous lines: smoothing function. 
Figure 8: Real-time observation of locked (left) and floating (right) lamellar eutectic patterns colliding at a eutectic-grain boundary during standard thin-sample directional solidification. The growth direction is directed vertically upward. a) $\mathrm{CBr}_{4}-\mathrm{C}_{2} \mathrm{Cl}_{6}$. Growth velocity: $\mathrm{V}=1.0 \mu \mathrm{ms}^{-1}$. The thicker lamellae are $\alpha$ and the thinner ones $\beta$. b) $\operatorname{In}-\operatorname{In}_{2} \mathrm{Bi}$. $\mathrm{V}=0.5 \mu \mathrm{ms}^{-1}$. The $\epsilon$ phase appears white and $\operatorname{In}_{2}$ Bi black. Bars: $20 \mu \mathrm{m}$.

Figure 9: Space-time map from Run RDS3. In- $\operatorname{In}_{2} \mathrm{Bi}$ eutectic. $\omega=2.5 \times 10^{-4} \mathrm{rad} \mathrm{s}^{-1}$. The direction of growth is clockwise. $\mathrm{R}_{1}, \mathrm{R}_{2}, \mathrm{R}_{3}$ : main angular ranges. $\mathrm{T}_{12}, \mathrm{~T}_{23}, \mathrm{~T}_{31}$ : transition angular ranges. $1 a$ to $3 b$ : different types of lamellar patterns. The radial cut is located in the $\mathrm{T}_{23}$ range on the left-hand side of the map. Image size: $2.4 \times 2.4 \mathrm{~mm}^{2}$.

Figure 10: Top: Snapshots taken at the indicated $\theta$-values within the same locking angular range. $\mathrm{CBr}_{4}-\mathrm{C}_{2} \mathrm{Cl}_{6}$ (Run RDS4). $\omega=5 \times 10^{-4} \mathrm{rad} \mathrm{s}^{-1}$. Horizontal dimension: 600 $\mu \mathrm{m}$. Bottom: $\phi(\theta)$ function. Symbols: measured values. Continuous line: best-fit linear function (slope $1 \pm 0.007$ ). The error bars are smaller than the symbol size.

Figure 11: Transition from a locking to a floating angular range during a RDS run. $\operatorname{In}_{-} \operatorname{In}_{2} \mathrm{Bi}$ eutectic (Run RDS5). $\omega=2.5 \times 10^{-4} \mathrm{rad} \mathrm{s}^{-1}$. Top: Space-time map. Arrow: growth direction. Straight lines: positions of the growth front at the indicated $\theta$-values. Vertical dimension: $340 \mu \mathrm{m}$. Bottom: Snapshot taken at $\theta=35^{\circ}$. Horizontal dimension: $300 \mu m$. 